\title{
MICORRIZA ARBUSCULAR E FERTILIZAÇÃO DO SOLO NO DESENVOLVIMENTO PÓS-TRANSPLANTE DE MUDAS DE SETE ESPÉCIES FLORESTAIS ${ }^{1}$
}

\author{
ENRIQUE POUYÚ-ROJAS² e JOSÉ OSWALDO SIQUEIRA ${ }^{3}$
}

RESUMO - Mudas de sete espécies florestais foram formadas em substrato de viveiro sem e com inoculação da mistura de fungos micorrízicos arbusculares (Glomus etunicatum, Gigaspora margarita e Acaulospora scrobiculata) e transplantadas para vasos com um solo Latossolo Vermelho-Escuro com alta e baixa fertilização com NPK e submetidas, ou não, a nova inoculação. Verificou-se, após 90 dias, que tanto a inoculação na formação quanto a inoculação no transplantio garantiu elevada colonização micorrízica $(>70 \%)$, estimulou o crescimento e aumentou os teores de alguns nutrientes nas plantas. Os efeitos no crescimento variaram entre as espécies e tratamentos, atingindo incrementos de matéria seca de até $800 \%$ em Colvillea racemosa. Plantas sem inoculação na formação e no transplantio, apresentaram crescimento reduzido, mesmo no solo com alta fertilidade, enquanto as plantas com inoculação na formação cresceram mais rapidamente, independentemente da reinoculação. A elevação da fertilidade não aumentou a matéria seca da parte aérea de Luehea grandiflora, Senna macranthera e Enterolobium contortisiliquum. Em Cecropia pachystachya aumentou apenas quando as mudas não foram submetidas a inoculação. Em Senna multijuga e em C. racemosa, a matéria seca da parte aérea aumentou quando as mudas foram submetidas a inoculação e em Sesbania virgata, aumentou em todos os tratamentos. Apenas C. racemosa não respondeu à inoculação no transplantio.

Termos para indexação: fungos micorrízicos, floresta tropical, adubação, reflorestamento, inoculação.

\section{ARBUSCULAR MYCORRHIZAL AND SOIL FERTILIZATION ON POST-TRANSPLANT DEVELOPMENT OF OUTPLANTS OF SEVEN FOREST SPECIES}

\begin{abstract}
Seedling of seven forest species were raised in conventional nursery substrate either infested or not with a mixture of arbuscular mycorrhizal fungi (Glomus etunicatum, Gigaspora margarita and Acaulospora scrobiculata) and transplanted into plastic pots with a low-fertility Oxisol amended with low or high NPK-fertilization and with or without re-inoculation. It was found that either inoculation at nursery or at transplanting stage guaranteed high root colonization $(>70 \%)$, growth response and favorable nutrition after transplant. Growth effects varied with treatments and with plant species. Inoculation benefits for shoot dry matter were as high as $800 \%$ for Colvillea racemosa. Reduced growth was observed when seedling non-inoculated at nursery stage were also not inoculated at transplanting, whereas nursery-inoculated ones grew well independently on soil fertility and re-inoculation. Enhanced soil fertility did not increase shoot dry matter yield of Luehea grandiflora, Senna macranthera and Enterolobium contortisiliquum. It increased dry matter of Cecropia pachystachya in the absence of mycorrhiza; of mycorrhizal Senna multijuga and C. racemosa and of Sesbania virgata caused an increase in all treatments. Only $C$. racemosa did not respond to inoculation at transplanting.
\end{abstract}

Index terms: mycorrhizal fungi, tropical forest, fertilization, reforestation, inoculation.

\footnotetext{
${ }^{1}$ Aceito para publicação em 19 de março de 1999.

Trabalho financiado pelo Convênio FAEPE/CEMIG e FAPEMIG.

${ }^{2}$ Eng. Agrôn., M.Sc., Instituto de Ecologia e Sistemática do Ministério de Ciência Tecnologia e Médio Ambiente de Cuba. Carr. Varona km 3 1/2. Capdevila Boyeros La Habana. AP-8010. Cuba. Bolsista do CNPq. E-mail: epouyu@ufla.br

${ }^{3}$ Eng. Agrôn., Ph.D., Prof. Titular, Dep. de Ciência do Solo, Universidade Federal de Lavras (UFLA), Caixa Postal 37, CEP 37200-000 Lavras, MG. Bolsista do CNPq. E-mail: siqueira@ufla.br
}

\section{INTRODUÇÃO}

Numerosos estudos têm sido desenvolvidos visando obter tecnologias para a reabilitação de solos degradados por atividades diversas e para a formação de matas ciliares, especialmente em áreas sob influência dos reservatórios de usinas hidrelétricas. Parte desses estudos compreende técnicas de revegetação com plantas nativas diversas, incluindo algumas com elevada dependência de fungos 
micorrízicos arbusculares (FMAs). Espécies micorrizo-dependentes devem ser submetidas a inoculação durante a formação das mudas, para garantir desenvolvimento adequado após seu plantio no campo (Herrera et al., 1988; Siqueira et al., 1993; Janos, 1996). Os FMAs associam-se às raízes das plantas, e propiciam a estas maior capacidade de competição em solos de baixa fertilidade, favorecendo a sobrevivência e desenvolvimento das plantas em condições adversas (Siqueira \& Saggin Júnior, 1995), tornando-se de grande importância para programas de restauração e reabilitação ambiental (Siqueira et al., 1994; Janos, 1996).

A prática da inoculação de espécies de FMAs selecionadas em mudas, além de favorecer o desenvolvimento destas, interfere na dinâmica do processo de sucessão da vegetação (Janos, 1996), facilitando a revegetação, especialmente em áreas com reduzido potencial de inóculo de FMAs. A inoculação dos FMAs nas mudas na formação é amplamente preconizada como maneira de viabilizar o uso dos FMAs, mas os efeitos desta prática no crescimento após o transplantio das mudas precisam ser melhor avaliados. No caso de espécies nativas, espera-se que, além da condição micorrízica das mudas, a existência de propágulos fúngicos e a fertilidade do solo onde estas serão plantadas exercerão grande influência no seu desenvolvimento. Siqueira et al. (1995) verificaram que o transplantio de mudas de cafeeiro sem micorrizas, para solo também isento de propágulos destes fungos, resultou em crescimento reduzido das mudas em comparação com as que foram submetidas a inoculação de fungos eficientes durante a formação. Considerando a importância dos FMAs para o reflorestamento com mudas de árvores nativas (Siqueira et al., 1998) é de grande interesse conhecer os efeitos dos fatores acima mencionados no crescimento pós-transplante de mudas destas espécies.

Avaliaram-se os efeitos da inoculação, nas plantas, da mistura de FMAs, durante a fase de formação das mudas e no ato do seu transplantio para solo isento de propágulos destes fungos, assim como os efeitos da fertilidade do solo, resultantes da adição de diferentes doses de NPK ou PK, no desenvolvimento pós-transplante em mudas de sete espécies florestais. As espécies foram selecionadas com base na disponibilidade de sementes e no interesse em plantios com fins conservacionistas, conforme indicações de Lorenzi (1992) e Davide et al. (1995), sendo estudadas as seguintes espécies: Enterolobium contortisiliquum (Vell.) Morong (tamboril), Luehea grandiflora Mart. et Zucc. (açoita-cavalo), Senna macranthera (Collard.) Irwin \& Barneby (fedegoso), Senna multijuga (L.C. Rich.) Irwin \& Barneby (cássiaverrugosa), Sesbania virgata (Caz.) Pers. (sesbânia), Cecropia pachystachya Trécul (embaúba) e Colvillea racemosa Bojer (colvílea).

\section{MATERIAL E MÉTODOS}

O estudo foi desenvolvido em casa de vegetação do Departamento de Ciência do Solo da Universidade Federal de Lavras (UFLA), de julho de 1995 até fevereiro de 1996. Mudas das sete espécies estudadas foram formadas com e sem inoculação de FMAs e transplantadas para solo em vasos contendo tratamentos de adubação quando foram, ou não, submetidas a reinoculação. O estudo constou de quatro tratamentos de inoculação e dois de adubação em delineamento experimental com parcelas subdivididas, com cinco repetições. Na parcela, consideraram-se tratamentos de fertilização, sendo duas doses de NPK ou apenas PK nas espécies nodulíferas, e na subparcela, quatro tratamentos com FMAs, a saber: (IF) inoculação apenas na formação da muda; (IT) inoculação apenas no transplantio da muda para o vaso; (IFT) inoculação nas duas épocas; (C) sem inoculação em nenhuma das épocas. Estes tratamentos foram testados separadamente em cada espécie vegetal, conforme detalhes apresentados a seguir.

\section{Formacão de mudas}

Sementes fornecidas pelo Departamento de Ciências Florestais-UFLA foram selecionadas, submetidas à quebra de dormência, conforme Davide et al. (1995), e germinadas em vermiculita, em bandejas com capacidade de 4 litros, cobertas com plástico transparente e irrigadas com água deionizada. Após a germinação, as plântulas foram repicadas para bandejas de polietileno com 96 tubetes com capacidade para $50 \mathrm{~mL}$ de substrato por tubete e submetidas a inoculação ou deixadas sem inoculação de FMAs e conduzidas por aproximadamente três meses. O substrato usado para a formação das mudas consistiu em $50 \%$ de Plantimax (Eucatex S/A), 20\% de húmus de minhoca, 20\% de solo de barranco, $10 \%$ de palha de arroz carbonizada, mistura esta suplementada com superfosfato simples $\left(1,5 \mathrm{~kg} \mathrm{~m}^{-3}\right)$ e fumigada com Bromex (brometo de metila) 
na dosagem de $393 \mathrm{~cm}^{3} \mathrm{~m}^{-3}$. Resultados de análises químicas parciais do substrato encontram-se na Tabela 1 .

Para a inoculação nas mudas em formação, realizouse a infestação do substrato com inóculo de FMAs, composto de uma mistura de Glomus etunicatum Becker \& Gerdemann, Gigaspora margarita Becker \& Hall e Acaulospora scrobiculata Trappe; com G. etunicatum representando $50 \%$ do total de esporos presentes no inóculo. O inóculo foi obtido de vaso de cultivo com Brachiaria decumbens, sendo este misturado ao substrato de modo a atingir uma densidade média final de quatro esporos $\mathrm{mL}^{-1}$ de substrato, além de outras formas de propágulos, como hifas e raízes colonizadas. Para equilibrar a microbiota entre os tratamentos inoculados e sem inoculação, adicionou-se aos últimos depois da repicagem das plântulas, $1 \mathrm{~mL}$ de filtrado do inóculo, isento de propágulos de FMAs Na sesbânia e no tamboril foram também inoculadas estirpes de rizóbio Br5401 e Br4406 (em turfa), provenientes da Embrapa-Centro Nacional de Pesquisa de Agrobiologia, em Seropédica, RJ. A inoculação de rizóbio, foi efetuada

TABELA 1. Características químicas do substrato e do solo antes da aplicação de calcário e nutrientes.

\begin{tabular}{lcc}
\hline \multirow{2}{*}{ Características } & \multicolumn{2}{c}{ Material utilizado } \\
\cline { 2 - 3 } & Substrato & Solo \\
\hline $\mathrm{pH} \mathrm{em} \mathrm{água}$ & 5,5 & 4,5 \\
$\mathrm{P}\left(\mathrm{mg} / \mathrm{dm}^{3}\right)^{2}$ & 101,0 & 1,0 \\
$\mathrm{~K}\left(\mathrm{mg} / \mathrm{dm}^{3}\right)^{3}$ & 107,0 & 30,0 \\
$\mathrm{~S}\left(\mathrm{mg} / \mathrm{dm}^{3}\right)^{4}$ & 123,3 & 3,8 \\
$\mathrm{Ca}\left(\mathrm{mmol}^{+} / \mathrm{dm}^{3}\right)^{5}$ & 40,9 & 6,0 \\
$\mathrm{Mg}\left(\mathrm{mmol}^{+} / \mathrm{dm}^{3}\right)^{5}$ & 32,6 & 2,0 \\
$\mathrm{Al}\left(\mathrm{mmol}^{+} / \mathrm{dm}^{3}\right)^{5}$ & 10,0 & 2,0 \\
$\mathrm{H}+\mathrm{Al}\left(\mathrm{mmol}^{+} / \mathrm{dm}^{3}\right)^{6}$ & 33,3 & 45,0 \\
$\mathrm{Zn}\left(\mathrm{mg} / \mathrm{dm}^{3}\right)^{7}$ & 6,5 & 0,5 \\
$\mathrm{Cu}\left(\mathrm{mg} / \mathrm{dm}^{3}\right)^{7}$ & 5,5 & 1,6 \\
$\mathrm{Fe}\left(\mathrm{mg} / \mathrm{dm}^{3}\right)^{7}$ & 73,2 & 76,6 \\
$\mathrm{Mn}\left(\mathrm{mg} / \mathrm{dm}^{3}\right)^{7}$ & 45,8 & 2,7 \\
$\mathrm{~B}\left(\mathrm{mg} / \mathrm{dm}^{3}\right)^{8}$ & 0,48 & 0,08 \\
$\mathrm{Matéria} \mathrm{orgânica}(\mathrm{g} / \mathrm{kg})^{9}$ & 54,0 & 27,0 \\
$\mathrm{~V}(\%)$ & - & 16,0 \\
\hline
\end{tabular}

1 Água $(1: 2,5) /$ potenciometria

2 Mehlich-I/colorimetria (Vettori, 1969).

3 Mehlich-I/fotometria de chama (Vettori, 1969).

4 Turbidimetria (Blanchar et al., 1965)

$5 \mathrm{KCl} 1,0 \mathrm{~mol} \mathrm{~L}^{-1 / \text { titulometria. }}$

${ }^{6} \mathrm{CaCl}_{2}$ 0,01 $\mathrm{mol} \mathrm{L}^{-1}(1: 2,5)+\mathrm{SMP} /$ potenciometria

7 DTPA/espectrofotometria de absorção atômica.

${ }^{8}$ Colorimétrico da curcumina.

9 Oxidação com dicromato de potássio. pela aplicação, do inoculante umedecido, às raízes das plântulas na repicagem para as bandejas. A partir de 30 dias após a repicagem, realizou-se adubação de cobertura com $\mathrm{N}(23,57 \mathrm{mM})$ na forma de $\left(\mathrm{NH}_{4}\right)_{2} \mathrm{SO}_{4} \mathrm{e}$ $\mathrm{K}(5,37 \mathrm{mM})$ como $\mathrm{KCl}$, repetida a cada três dias até o transplantio das mudas. Nas espécies nodulíferas aplicouse apenas K. Aos 90 dias, avaliaram-se o crescimento em altura, colonização micorrízica de todas as espécies, e a nodulação no tamboril e na sesbânia, utilizando-se dez mudas por tratamento. A altura das plantas nesta época corresponde às alturas da época 0 da Tabela 2 . As mudas do substrato infestado com propágulos de FMAs apresentaram colonização micorrízica elevada, superior a $80 \%$ em todas as espécies, sendo denominadas infectadas durante a formação (IF), enquanto as formadas em substrato não-infestado não apresentaram sinais de colonização, e foram designadas controle $(\mathrm{C})$, sem inoculação na formação. O número de nódulos por planta foi, em média, $61 \pm 18$ e $59 \pm 17 ; 5 \pm 3$ e $2 \pm 1$ na sesbânia e tamboril, com e sem inoculação, respectivamente. Para o transplante, as mudas foram padronizadas quanto ao vigor e desenvolvimento.

\section{Mudas transplantadas para vasos com solo}

Utilizou-se solo superficial (0-20 $\mathrm{cm}$ de profundidade), coletado de um Latossolo Vermelho-Escuro (LE), proveniente de área sob vegetação de campo, próxima à represa de Camargos, na região dos Campos das Vertentes, em Minas Gerais. Solo secado ao ar e peneirado em malha de $4 \mathrm{~mm}$ (análise química parcial, Tabela 1), recebeu calagem com calcário dolomítico para elevar $\mathrm{V}_{2}$ para $60 \%$ (Raij, 1991). Após a calagem, o solo foi fumigado, aplicando-se $393 \mathrm{~cm}^{3}$ de Bromex (Brometo de metila 98\% + Cloropicrina $2 \%$ ) por $\mathrm{m}^{3}$ de solo, em caixa de alvenaria vedada, durante 48 horas, e deixado mais 48 horas em ar livre para a exalação do excesso de gases. Em seguida colocaram-se $3 \mathrm{~kg}$ deste solo em cada vaso onde foram aplicados os tratamentos de fertilização, realizados mediante aplicação diferenciada de doses de NPK. Na dose baixa $\left(\mathrm{D}_{1}\right)$, aplicaram-se 25,25 e $83 \mathrm{mg} \mathrm{kg}^{-1}$ de solo, de $\mathrm{N}, \mathrm{P}_{2} \mathrm{O}_{5}$ e $\mathrm{K}_{2} \mathrm{O}$ respectivamente, enquanto na dose alta $\left(D_{2}\right)$ estes valores foram quadruplicados. Nas espécies nodulíferas omitiu-se a aplicação do N. Análises de solo antes do transplantio revelaram 2 e 55; 19 e $92 \mathrm{mg} \mathrm{kg}^{-1} \mathrm{de}$ solo de $\mathrm{P}$ e $\mathrm{K}$, respectivamente, para as condições de baixa e alta fertilização. Utilizaram-se sulfato de amônia $(20 \%$ de $\mathrm{N})$, superfosfato simples $\left(18 \%\right.$ de $\left.\mathrm{P}_{2} \mathrm{O}_{5}\right)$ e cloreto de potássio $\left(60 \%\right.$ de $\left.\mathrm{K}_{2} \mathrm{O}\right)$ como fontes de nutrientes. Os tratamentos com inóculo no transplantio (IT) foram realizados pela aplicação de $10 \mathrm{~mL}$ de inóculo, o mesmo utilizado no período de formação, contendo, em média, 
27 esporos por $\mathrm{mL}$ (270 esporos/planta). O experimento foi conduzido por 100 dias, após a repicagem, quando se avaliaram a colonização micorrízica, área foliar, matéria seca da parte aérea e raízes, teores de nutrientes, e atividade da nitrogenase nos nódulos.
A altura das plantas foi avaliada aos 30,60 e 90 dias do transplantio. Para avaliação da área foliar empregouse aparelho medidor de área, modelo LI-3000A (LI-COR, Lincoln, U.S.A). A parte aérea das plantas foi cortada, lavada com água destilada, e secada em estufa a $60^{\circ} \mathrm{C}$, até

TABELA2. Altura de plantas (cm), aos 30, 60 e 90 dias após o transplantio, de espécies arbóreas sob tratamentos de inoculação e doses baixa $\left(D_{1}\right)$ e alta $\left(D_{2}\right)$ de macronutrientes aplicados no solo ${ }^{1}$.

\begin{tabular}{|c|c|c|c|c|c|c|c|c|c|c|}
\hline \multirow{2}{*}{$\begin{array}{l}\text { Trata- } \\
\text { mento }^{2}\end{array}$} & \multicolumn{2}{|c|}{0} & \multicolumn{2}{|c|}{30} & \multicolumn{2}{|c|}{60} & \multicolumn{2}{|c|}{90} & \multicolumn{2}{|c|}{ Regressão $^{3} / \mathrm{R}^{2}$} \\
\hline & $\mathrm{D}_{1}$ & $\mathrm{D}_{2}$ & $\mathrm{D}_{1}$ & $\mathrm{D}_{2}$ & $D_{1}$ & $\mathrm{D}_{2}$ & $\mathrm{D}_{1}$ & $\mathrm{D}_{2}$ & $\mathrm{D}_{1}$ & $\mathrm{D}_{2}$ \\
\hline & \multicolumn{10}{|c|}{ Açoita-cavalo } \\
\hline IF & $17,5 \mathrm{a}$ & $17,5 \mathrm{a}$ & $20,0 \mathrm{a}$ & $23,3 \mathrm{a}$ & $32,5 \mathrm{a}$ & $34,5 \mathrm{a}$ & $37,4 \mathrm{a}$ & $41,6 \mathrm{a}$ & $\mathrm{L}, 0,97 * * *$ & $\mathrm{~L}, 0,98 * * *$ \\
\hline IT & $18,0 \mathrm{a}$ & $18,0 \mathrm{a}$ & $23,2 \mathrm{a}$ & $22,6 \mathrm{a}$ & $35,9 a$ & $36,7 \mathrm{a}$ & $43,0 \mathrm{a}$ & $40,8 \mathrm{a}$ & $\mathrm{L}, 0,93 * * *$ & $\mathrm{~L}, 0,94 * * *$ \\
\hline IFT & $17,5 \mathrm{a}$ & $17,5 \mathrm{a}$ & $20,4 a$ & $21,2 \mathrm{a}$ & $33,9 a$ & $35,8 \mathrm{a}$ & $37,8 \mathrm{a}$ & $40,5 a$ & $\mathrm{~L}, 0,93 * * *$ & $\mathrm{~L}, 0,93 * * *$ \\
\hline \multirow[t]{2}{*}{$\mathrm{C}$} & $18,0 \mathrm{a}$ & $18,0 \mathrm{a}$ & $19,2 \mathrm{a}$ & $21,2 \mathrm{a}$ & $25,0 \mathrm{a}$ & $31,2 \mathrm{a}$ & $26,0 \mathrm{~b}$ & $33,8 b$ & $\mathrm{~L}, 0,91 * * *$ & $\mathrm{~L}, 0,94 * * *$ \\
\hline & \multicolumn{10}{|c|}{ Cássia-verrugosa } \\
\hline IF & $14,8 \mathrm{a}$ & $14,8 \mathrm{a}$ & $22,1 \mathrm{ab}$ & $25,1 \mathrm{a}$ & $30,9 \mathrm{ab}$ & $48,4 a^{*}$ & $47,2 \mathrm{a}$ & $65,8 a^{*}$ & $\mathrm{Q}, 0,96 * * *$ & $\mathrm{~L}, 0,98 * * *$ \\
\hline IT & $12,9 \mathrm{a}$ & $12,9 \mathrm{a}$ & $25,5 \mathrm{a}$ & $23,8 \mathrm{a}$ & $37,0 \mathrm{a}$ & $43,3 a^{*}$ & $57,2 \mathrm{a}$ & $64,0 \mathrm{a}$ & $\mathrm{Q}, 0,97 * * *$ & $\mathrm{~L}, 0,98 * * *$ \\
\hline IFT & $14,8 \mathrm{a}$ & $14,8 \mathrm{a}$ & $24,6 a b^{*}$ & $20,9 a b$ & $31,2 \mathrm{ab}$ & $34,2 b$ & $50,2 \mathrm{a}$ & $47,8 b$ & $\mathrm{Q}, 0,96 * * *$ & $\mathrm{~L}, 0,98^{* * *}$ \\
\hline \multirow[t]{2}{*}{$\mathrm{C}$} & $12,9 \mathrm{a}$ & $12,9 \mathrm{a}$ & $20,4 \mathrm{~b}$ & $18,1 \mathrm{~b}$ & $24,8 b$ & $23,5 b$ & $29,2 b$ & $27,8 b$ & $\mathrm{~L}, 0,99 * * *$ & $\mathrm{~L}, 0,99 * * *$ \\
\hline & \multicolumn{10}{|c|}{ Colvílea } \\
\hline IF & $8,4 a$ & $8,4 \mathrm{a}$ & $10,5 \mathrm{a}$ & $10,5 \mathrm{a}$ & $12,8 \mathrm{a}$ & $14,0 \mathrm{a}$ & $13,2 \mathrm{a}$ & $16,0 a^{*}$ & $\mathrm{~L}, 0,93 * * *$ & $\mathrm{Q}, 0,98 * * *$ \\
\hline IT & $7,7 \mathrm{a}$ & $7,7 \mathrm{a}$ & $8,2 \mathrm{a}$ & $9,9 \mathrm{a}$ & $10,4 \mathrm{ab}$ & $10,5 b$ & $10,6 b c$ & $10,9 b$ & $\mathrm{~L}, 0,89 * * *$ & $\mathrm{Q}, 0,85^{* * *}$ \\
\hline IFT & $8,4 \mathrm{a}$ & $8,4 \mathrm{a}$ & $9,6 \mathrm{a}$ & $11,1 \mathrm{a}$ & $11,7 \mathrm{ab}$ & $13,5 \mathrm{a}$ & $11,8 \mathrm{ab}$ & $14,6 a^{*}$ & $\mathrm{~L}, 0,91 * * *$ & $\mathrm{Q}, 0,96^{* * * *}$ \\
\hline \multirow[t]{2}{*}{$\mathrm{C}$} & $7,7 \mathrm{a}$ & $7,7 \mathrm{a}$ & $8,0 \mathrm{a}$ & $8,7 \mathrm{a}$ & $9,4 b$ & $10,0 b$ & $9,6 \mathrm{c}$ & $10,4 \mathrm{~b}$ & $\mathrm{~L}, 0,90 * * *$ & $\mathrm{Q}, 0,96^{* * *}$ \\
\hline & \multicolumn{10}{|c|}{ Embaúba } \\
\hline IF & $16,3 \mathrm{a}$ & $16,3 \mathrm{a}$ & $21,2 \mathrm{a}$ & $21,2 \mathrm{a}$ & $32,8 \mathrm{a}$ & $32,0 \mathrm{a}$ & $39,4 \mathrm{a}$ & $39,2 \mathrm{a}$ & $\mathrm{L}, 0,89 * * *$ & $\mathrm{~L}, 0,98 * * *$ \\
\hline IT & $16,0 \mathrm{a}$ & $16,0 \mathrm{a}$ & $24,1 \mathrm{a}$ & $20,8 \mathrm{a}$ & $33,6 \mathrm{a}$ & $36,9 a$ & $40,0 \mathrm{a}$ & $39,5 \mathrm{a}$ & $\mathrm{L}, 0,91 * * *$ & $\mathrm{~L}, 0,98 * * *$ \\
\hline IFT & $16,3 \mathrm{a}$ & $16,3 \mathrm{a}$ & $24,1 \mathrm{a}$ & $22,8 \mathrm{a}$ & $33,6 \mathrm{a}$ & $37,4 \mathrm{a}$ & $37,9 \mathrm{a}$ & $43,6 \mathrm{a}$ & $\mathrm{L}, 0,93 * * *$ & $\mathrm{~L}, 0,98 * * *$ \\
\hline \multirow[t]{2}{*}{$\mathrm{C}$} & $16,0 \mathrm{a}$ & $16,0 \mathrm{a}$ & $17,8 \mathrm{a}$ & $20,5 a$ & $27,1 \mathrm{a}$ & $30,2 \mathrm{a}$ & $29,8 \mathrm{a}$ & $36,2 \mathrm{a}$ & $\mathrm{L}, 0,92 * * *$ & $\mathrm{~L}, 0,99 * * *$ \\
\hline & \multicolumn{10}{|c|}{ Fedegoso } \\
\hline IF & $15,4 \mathrm{a}$ & $15,4 \mathrm{a}$ & $22,8 \mathrm{a}$ & $20,2 \mathrm{a}$ & $30,8 \mathrm{a}$ & $33,2 \mathrm{a}$ & $35,2 \mathrm{a}$ & $35,2 \mathrm{a}$ & $\mathrm{L}, 0,97 * * *$ & $\mathrm{~L}, 0,97 * * *$ \\
\hline IT & $13,2 \mathrm{a}$ & $13,2 \mathrm{a}$ & $23,1 \mathrm{a}$ & $21,0 \mathrm{a}$ & $32,3 a$ & $30,0 \mathrm{a}$ & $34,5 \mathrm{a}$ & $34,5 \mathrm{a}$ & $\mathrm{L}, 0,98 * * *$ & $\mathrm{~L}, 0,98 * * *$ \\
\hline IFT & $15,4 \mathrm{a}$ & $15,4 \mathrm{a}$ & $24,6 a$ & $20,6 a$ & $32,8 \mathrm{a}$ & $28,8 \mathrm{a}$ & $34,2 \mathrm{a}$ & $34,2 \mathrm{a}$ & $\mathrm{L}, 0,98 * * *$ & $\mathrm{~L}, 0,99 * * *$ \\
\hline \multirow[t]{2}{*}{$\mathrm{C}$} & $13,2 \mathrm{a}$ & $13,2 \mathrm{a}$ & $13,4 \mathrm{a}$ & $16,1 \mathrm{a}$ & $14,3 \mathrm{a}$ & $18,0 \mathrm{a}$ & $21,0 \mathrm{~b}$ & $21,0 \mathrm{~b}$ & $\mathrm{~L}, 0,72 * *$ & $\mathrm{~L}, 0,95 * * *$ \\
\hline & \multicolumn{10}{|c|}{ Sesbânia } \\
\hline IF & $29,6 \mathrm{a}$ & $29,6 \mathrm{a}$ & $40,3 a$ & $36,4 a$ & $58,8 \mathrm{a}$ & $63,6 b$ & $59,0 \mathrm{a}$ & $64,8 a^{*}$ & $\mathrm{~L}, 0,90 * * *$ & $\mathrm{~L}, 0,89 * * *$ \\
\hline IT & $30,4 a$ & $30,4 \mathrm{a}$ & $42,3 a$ & $40,5 \mathrm{a}$ & $60,8 \mathrm{a}$ & $71,0 a^{*}$ & $61,6 a$ & $71,2 a^{*}$ & $\mathrm{~L}, 0,91 * * *$ & $\mathrm{~L}, 0,92 * * *$ \\
\hline IFT & $29,6 a$ & $29,6 \mathrm{a}$ & $39,5 a$ & $39,1 \mathrm{a}$ & $58,0 \mathrm{a}$ & $64,6 a b^{*}$ & $57,6 a$ & $69,0 a^{*}$ & $\mathrm{~L}, 0,89 * * *$ & $\mathrm{~L}, 0,93 * * *$ \\
\hline $\mathrm{C}$ & $30,4 a$ & $30,4 \mathrm{a}$ & $33,3 b$ & $40,2 a^{*}$ & $44,4 b$ & $59,4 b^{*}$ & $44,0 \mathrm{~b}$ & $60,2 a^{*}$ & $\mathrm{~L}, 0,86^{* * *}$ & $\mathrm{~L}, 0,90 * * *$ \\
\hline & \multicolumn{10}{|c|}{ Tamboril } \\
\hline IF & $20,8 \mathrm{a}$ & $20,8 \mathrm{a}$ & $20,8 \mathrm{a}$ & $28,5 \mathrm{a}$ & $34,2 \mathrm{a}$ & $44,8 a b^{*}$ & $37,3 \mathrm{a}$ & $48,6 a^{*}$ & $\mathrm{~L}, 0,87 * * *$ & $\mathrm{~L}, 0,89 * * *$ \\
\hline IT & $20,0 \mathrm{a}$ & $20,0 \mathrm{a}$ & $22,4 a$ & $22,2 b$ & $28,4 \mathrm{a}$ & $55,0 a^{*}$ & $36,0 \mathrm{a}$ & $58,8 \mathrm{a}^{*}$ & $\mathrm{~L}, 0,89 * * *$ & $\mathrm{~L}, 0,89 * * *$ \\
\hline IFT & $20,8 \mathrm{a}$ & $20,8 \mathrm{a}$ & $20,8 \mathrm{a}$ & $22,9 b$ & $32,0 \mathrm{a}$ & $40,4 b$ & $32,8 \mathrm{a}$ & $42,4 \mathrm{ab}$ & $\mathrm{L}, 0,89 * * *$ & $\mathrm{~L}, 0,87 * * *$ \\
\hline $\mathrm{C}$ & $20,0 \mathrm{a}$ & $20,0 \mathrm{a}$ & $20,0 \mathrm{a}$ & $20,0 \mathrm{c}$ & $23,0 \mathrm{a}$ & $25,2 \mathrm{c}$ & $27,0 \mathrm{a}$ & $25,8 \mathrm{~b}$ & $\mathrm{~L}, 0,91 * *$ & $\mathrm{Q}, 0,91 * * *$ \\
\hline
\end{tabular}

${ }^{1}$ Médias seguidas da mesma letra não diferem entre si em tratamentos de inoculação em cada espécie pelo teste de Tukey a $5 \%$ de probabilidade.

2 IF: plantas com inoculação na formação das mudas; IT: plantas com inoculação no transplante; IFT: plantas com inoculação nas duas épocas; C: plantas sem nenhuma inoculação.

${ }^{3}$ L: regressão linear; Q: regressão quadrática.

* Efeito significativo de doses de fertilização a $5 \%$ de probabilidade

$* *$ e *** Significativo a $5 \%$ e $1 \%$ de probabilidade, respectivamente 
atingir peso constante. Das raízes retiradas do solo e lavadas, coletou-se $1 \mathrm{~g}$ de raízes finas que foram armazenadas em FAA(Formalina-álcool-ácido acético), para avaliação posterior da colonização micorrízica após a clarificação e coloração com azul de tripano (Phillips \& Hayman, 1970) e determinação conforme Giovannetti \& Mosse (1980). Nas espécies nodulíferas, determinaram-se o número e o peso de nódulos e a atividade da nitrogenase, pela técnica da redução do acetileno. Para isto, foram retirados, de cada planta, cinco nódulos, que foram colocados em frasco de vidro de $5 \mathrm{~mL}$, fechado hermeticamente. Dada a nodulação reduzida nas plantas sem inoculação, avaliaram-se os nódulos existentes em cada repetição. Em seguida, injetouse $1 \mathrm{~mL}$ de $\mathrm{C}_{2} \mathrm{H}_{2}$ em cada frasco e incubou-se durante uma hora, e então uma amostra de $1 \mathrm{~cm}^{3}$ foi retirada com seringa, e injetada em cromatógrafo de gás (VARIANT-Star $3400 \mathrm{Cx}$ ), para a determinação do $\mathrm{C}_{2} \mathrm{H}_{4}$ (etileno) produzido. Os resultados da atividade da nitrogenase foram expressos em $\mu$ moles de $\mathrm{C}_{2} \mathrm{H}_{4} \mathrm{~g}^{-1}$ de nódulos frescos hora ${ }^{-1}$. As raízes e parte aérea secas foram pesadas, moídas e utilizadas, para determinação dos teores de nutrientes. $\mathrm{O} \mathrm{N}$ foi determinado pelo método semi-micro Kjeldahl (Liao, 1981), e a destilação e titulação, segundo Bremner \& Edward (1965). No extrato obtido por digestão nitroperclórica segundo Zarosky \& Burau (1977), determinaram-se os teores de $\mathrm{Ca}$ e $\mathrm{Zn}$ por espectrofotometria de absorção atômica; $\mathrm{P}$, por fotocolorimetria, e $\mathrm{K}$, por fotometria de chama.

Todos os dados foram submetidos à análise de variância e a testes de médias, utilizando-se o programa estatístico SANEST (Zonta et al., 1984). Os dados de colonização micorrízica e de nodulação foram transformados por $\operatorname{arcsen}(\mathrm{x} / 100)^{1 / 2} \mathrm{e}(\mathrm{x}+0,5)^{1 / 2}$, respectivamente

\section{RESULTADOS E DISCUSSÃO}

\section{Altura das plantas em diferentes épocas}

O crescimento pós-transplante das espécies, avaliado pela altura das plantas em diferentes épocas, foi muito influenciado pelos tratamentos (Tabela 2), e seus efeitos, diferenciados nas espécies. No açoita-cavalo, verificou-se uma resposta linear em função do tempo, sendo a altura das plantas, aos 90 dias, menor no controle sem inoculação (C) do que nos demais tratamentos de inoculação, em ambas as doses de NPK. A elevação na dose de NPK não influenciou o crescimento desta espécie. Comportamento idêntico da inoculação foi observado no fedegoso, que apresentou resposta também linear quanto ao crescimento nas duas doses de NPK. A embaúba também apresentou resposta linear em épocas de avaliação, porém não foi influenciada significativamente pelos tratamentos de inoculação. No entanto, plantas controle na baixa fertilização eram em torno de $25 \%$ menores que as inoculadas. A cássia-verrugosa mostrou diferenças no crescimento já aos 30 dias do transplantio. Estas diferenças aumentaram com o tempo, e aos 90 dias as plantascontrole eram menores que as de todos os tratamentos que receberam inoculação na dose baixa de NPK. Na dose alta, plantas C e IFT não diferiram entre si, mas cresceram menos que as IF e IT. A fertilização reduziu o crescimento das plantas IFT aos 30 dias, e favoreceu as IF e IT aos 60, e as IF, aos 90 dias, quando as plantas deste tratamento atingiram crescimento máximo na dose mais elevada de NPK. Esta espécie apresentou crescimento segundo um modelo quadrático na dose baixa, exceto no que tange ao controle, e linear na dose alta de NPK. A sesbânia exibiu resposta linear quanto ao crescimento, em função da época, em todos os tratamentos, e já mostrava resposta à inoculação aos 30 dias na baixa fertilização. Estas respostas permaneceram nas épocas posteriores, desaparecendo na dose mais alta de PK aos 90 dias, quando não havia diferenças entre os tratamentos de inoculação. O tamboril, no solo com fertilização alta, exibiu diferenças marcantes entre os tratamentos de inoculação, já aos 30 dias. Plantas sem inoculação eram menores que as IT e IFT as quais eram menores que as IF. Na dose baixa de PK não houve diferença entre os tratamentos, em nenhuma das épocas de avaliação. Aos 90 dias, plantas IT apresentaram altura média máxima $(58,8 \mathrm{~cm})$, sem, no entanto, diferir estatisticamente das IF $(48,6 \mathrm{~cm})$. As plantas-controle cresceram muito pouco (média $=25,8 \mathrm{~cm}$ de altura), mesmo quando bem adubadas. Verifica-se uma ação aditiva da inoculação e adubação nesta espécie, como relatado para o cafeeiro (Siqueira et al., 1993) e essências nativas (Carneiro et al., 1996). A colvílea mostrou diferença em relação à inoculação aos 60 e 90 dias, quando plantas sem inoculação no período da formação apresentaram menores alturas que as dos demais tratamentos, na maior fertilização. Portanto, nesta espécie, a inoculação na época do transplantio não foi eficaz para o crescimento. Resposta à elevação da aduba- 
ção só foi verificada aos 90 dias nas plantas com inoculação na época da formação. O crescimento seguiu modelo linear em todos os tratamentos de inoculação e adubação.

Verificou-se que, dependendo da fertilidade do solo, todas as espécies tiveram crescimento póstransplantio reduzido, quando não foram submetidas a inoculação. Deve-se ressaltar que no tocante à embaúba esta diferença não alcançou significância $(\mathrm{P} \leq 0,05)$ neste estudo, contrariando sua alta responsividade aos FMAs (Carneiro et al., 1996; Saggin Júnior, 1997; Siqueira et al., 1998). Apenas a colvílea não respondeu à inoculação na época do transplantio, resultado que sugere a necessidade de estudos adicionais. A elevação da fertilidade do solo pode compensar, pelo menos em algumas espécies, a ausência de micorrizas, o que já foi constatado no cafeeiro (Saggin Júnior et al., 1994). O crescimento e resposta da sesbânia à inoculação mostrou-se muito dependente da fertilidade, corroborando os resultados de Moreira \& Siqueira (1995), em solo de baixa fertilidade.

\section{Colonização micorrízica, área foliar e matéria seca}

Ao contrário do que foi observado no que se refere a plantas-controle, nas plantas com inoculação no período da formação (IF) e na época do transplantio (IT) houve colonização micorrízica (CM) elevada. Os altos valores de CM e os efeitos positivos da inoculação em ambas as épocas sugerem que qualquer um dos procedimentos de inoculação empregados garante adequada CM das sete espécies estudadas (Tabela 3). A elevação na fertilização exerceu efeito adverso significativo na CM em apenas quatro espécies, sendo este efeito muito pequeno e diferenciado, em razão da época da inoculação. Ainda assim, a CM manteve-se elevada, o que explica os benefícios da inoculação para o crescimento destas espécies mesmo na alta fertilização. Por se tratar de um solo pobre, as quantidades de nutrientes aplicadas não foram suficientes para eliminar os benefícios das micorrizas no período estudado. O fato de o açoita-cavalo e a embaúba mostrarem-se indiferentes à fertilização pode resultar dos efeitos benéficos dos FMAs para essas plantas, considerando sua alta dependência micorrízica (Saggin Júnior, 1997). A colvílea, apesar da elevada colonização obtida, foi a única espécie que não respondeu à inoculação na época do transplantio. Os resultados aqui apresentados, corroborando os referentes ao cafeeiro (Saggin Júnior et al., 1992; Siqueira et al., 1995), evidenciam a importância da condição micorrízica do solo para onde as mudas sem inoculação prévia serão transplantadas.

Os tratamentos de inoculação e de adubação exerceram efeitos diferenciados nos parâmetros vegetativos das espécies (Tabela 3). Verificou-se maior área foliar (AF) em diversos tratamentos de inoculação na alta fertilização, sendo este efeito mais acentuado no tamboril e cássia-verrugosa. Fato interessante é observado na colvílea, que apesar da colonização elevada obtida com a inoculação no transplantio (IT) de plantas sem inoculação no período da formação, não houve aumento de $\mathrm{AF}$. Entretanto, plantas que receberam inóculo no período de formação (IF) tiveram incrementos de AF de até $900 \%$, em relação às sem nenhuma inoculação, as quais apresentaram AF muito reduzida. $\mathrm{Na}$ sesbânia, que cresceu com alta fertilização, não houve diferença entre plantas sem inoculação e as IF ou IFT. Nesta espécie, na embaúba e açoita-cavalo, a elevação da fertilização resultou também em aumento na $\mathrm{AF}$ das plantas sem inoculação no período da formação, indicando a importância dos nutrientes para o desenvolvimento inicial quando mudas destas espécies não são micorrizadas.

Quanto ao peso de matéria seca da parte aérea, apenas a sesbânia não mostrou efeito da micorrização, enquanto o açoita-cavalo, o fedegoso e o tamboril não responderam à elevação da dose de nutrientes aplicados no solo (Tabela 3). Na colvílea, tal como ocorreu na área foliar, a inoculação na época da formação das mudas promoveu incrementos de 700 e $1.250 \%$ nas doses baixa $\left(D_{1}\right)$ e alta $\left(D_{2}\right)$ de fertilização, respectivamente, quando comparadas com as mudas não inoculadas nesta época. É interessante ressaltar o fato de que a resposta à fertilização só foi observada nas plantas IF, e que a inoculação na época do transplantio (IT), embora tenha garantido elevada colonização (82 e 91\%), não resultou em benefícios para a produção de matéria seca desta espécie. Na embaúba, a maior produção de matéria seca da parte aérea, foi obtida em plantas 
submetidas a inoculação nas duas épocas (IFT), não diferindo no tocante à fertilização. Nesta mesma dose, plantas IT produziram mais matéria seca que as IF, o que indica efeito aditivo da IT com a elevação da fertilidade do solo. A sesbânia mostrou notável resposta à elevação da fertilização, especialmente as plantas-controle, sem, entretanto, haver diferenças entre os tratamentos de inoculação. Nesta espécie, a elevação da fertilidade do solo pode compensar a ausência de micorrizas, fato não verificado para colvílea.

A produção de matéria seca de raízes foi também influenciada pelos tratamentos (Tabela 3). Em açoita-cavalo, embaúba e sesbânia, não houve diferenças em tratamentos de inoculação na dose mais elevada de nutrientes, ao contrário do que foi observado em todas as espécies em condições de fertilidade baixa. Portanto, são evidentes os benefícios da

TABELA3. Colonização micorrízica (CM), área foliar (AF), matéria seca da parte aérea (MSPA) e raízes (MSR) de espécies arbóreas sob tratamentos de inoculação e doses baixa $\left(D_{1}\right)$ e alta $\left(D_{2}\right)$ de nutrientes aplicados no solo'.

\begin{tabular}{|c|c|c|c|c|c|c|c|c|c|}
\hline \multirow[t]{2}{*}{ Espécie } & \multirow{2}{*}{$\begin{array}{l}\text { Trata- } \\
\text { mento }^{2}\end{array}$} & \multicolumn{2}{|c|}{$\mathrm{CM}$} & \multicolumn{2}{|c|}{$\mathrm{AF}$} & \multicolumn{2}{|c|}{ MSPA } & \multicolumn{2}{|c|}{ MSR } \\
\hline & & $\mathrm{D}_{1}$ & $\mathrm{D}_{2}$ & $\mathrm{D}_{1}$ & $\mathrm{D}_{2}$ & $\mathrm{D}_{1}$ & $\mathrm{D}_{2}$ & $\mathrm{D}_{1}$ & $\mathrm{D}_{2}$ \\
\hline \multirow{5}{*}{ Açoita-cavalo } & & $\begin{array}{l}------ \\
---\end{array}$ & $\begin{array}{l}----- \\
-\cdots\end{array}$ & $---\left(\mathrm{cm}^{2}\right.$ & anta $\left.^{-1}\right)---$ & $---(\mathrm{g} \mathrm{p}$ & $\left.\mathrm{ta}^{-1}\right)-{ }^{--}$ & $--\left(g_{1}\right.$ & $\left(\mathrm{ta}^{-1}\right)--$ \\
\hline & IF & $74 a$ & $74 \mathrm{a}$ & $761 \mathrm{ab}$ & $589 \mathrm{ab}$ & $6,1 \mathrm{a}$ & $7,4 \mathrm{a}$ & $3,6 a b$ & $3,0 \mathrm{a}$ \\
\hline & IT & $80 \mathrm{a}$ & $79 a$ & $850 \mathrm{a}$ & $835 \mathrm{a}$ & $8,6 a$ & $8,9 a$ & $4,8 \mathrm{a}^{*}$ & $3,4 \mathrm{a}$ \\
\hline & IFT & $85 \mathrm{a}$ & $86 a$ & $370 \mathrm{bc}$ & 631ab* & $7,1 \mathrm{ab}$ & $8,7 \mathrm{a}$ & $3,2 b$ & $2,9 \mathrm{a}$ \\
\hline & $\mathrm{C}$ & $0 \mathrm{~b}$ & $0 \mathrm{~b}$ & $150 \mathrm{c}$ & $398 b^{*}$ & $3,7 \mathrm{~b}$ & $4,2 b$ & $1,5 \mathrm{c}$ & $4,1 a^{*}$ \\
\hline \multirow[t]{4}{*}{ C. verrugosa } & IF & $88 a^{*}$ & $70 \mathrm{~b}$ & $294 a$ & $416 a^{*}$ & $8,4 \mathrm{a}$ & $12,0 a^{*}$ & $6,2 \mathrm{a}$ & $9,8 a^{*}$ \\
\hline & IT & $81 \mathrm{a}$ & $81 \mathrm{ab}$ & $332 \mathrm{a}$ & $387 \mathrm{a}$ & $8,8 \mathrm{a}$ & $9,9 b$ & $1,8 b$ & $6,9 a b^{*}$ \\
\hline & IFT & $85 \mathrm{a}$ & $88 \mathrm{a}$ & $329 a$ & $339 a$ & $9,2 \mathrm{a}$ & $9,4 b$ & $5,9 \mathrm{a}$ & $5,9 \mathrm{~b}$ \\
\hline & $\mathrm{C}$ & $0 \mathrm{~b}$ & $0 \mathrm{c}$ & $81 \mathrm{~b}$ & $67 \mathrm{~b}$ & $2,1 \mathrm{c}$ & $2,0 \mathrm{c}$ & $8,7 a^{*}$ & $2,8 \mathrm{c}$ \\
\hline \multirow[t]{4}{*}{ Colvílea } & IF & $82 \mathrm{a}$ & $79 a$ & $308 \mathrm{a}$ & $486 a^{*}$ & $1,7 \mathrm{a}$ & $2,7 a^{*}$ & $2,1 \mathrm{a}$ & $3,0 a^{*}$ \\
\hline & IT & $91 a^{*}$ & $82 \mathrm{a}$ & $29 \mathrm{~b}$ & $42 b$ & $0,2 \mathrm{c}$ & $0,2 \mathrm{~b}$ & $0,6 b$ & $0,3 \mathrm{~b}$ \\
\hline & IFT & $85 a$ & $86 a$ & $219 \mathrm{a}$ & $435 a^{*}$ & $1,0 \mathrm{~b}$ & $2,4 a^{*}$ & $0,9 b$ & $2,7 a^{*}$ \\
\hline & $\mathrm{C}$ & $0 \mathrm{~b}$ & $0 \mathrm{~b}$ & $51 b$ & $49 b$ & $0,3 \mathrm{c}$ & $0,3 \mathrm{~b}$ & $0,4 b$ & $0,4 \mathrm{~b}$ \\
\hline \multirow[t]{4}{*}{ Embaúba } & IF & $86 a$ & $83 a$ & $857 \mathrm{a}$ & $606 a$ & $4,5 \mathrm{ab}$ & $3,8 \mathrm{~b}$ & $5,4 \mathrm{a}$ & $4,1 \mathrm{a}$ \\
\hline & IT & $79 a$ & $76 a$ & $749 a$ & $901 \mathrm{a}$ & $4,2 b$ & $4,4 \mathrm{ab}$ & $5,3 a$ & $5,4 \mathrm{a}$ \\
\hline & IFT & $82 \mathrm{a}$ & $87 a$ & $797 a$ & $933 a$ & $6,4 \mathrm{a}$ & $6,2 \mathrm{a}$ & $4,6 a$ & $4,8 \mathrm{a}$ \\
\hline & $\mathrm{C}$ & $0 \mathrm{~b}$ & $0 \mathrm{~b}$ & $151 b$ & $488 b^{*}$ & $0,5 \mathrm{c}$ & $2,5 b^{*}$ & $2,5 b$ & $4,9 a^{*}$ \\
\hline \multirow[t]{4}{*}{ Fedegoso } & IF & $93 a$ & $99 a$ & $958 \mathrm{a}$ & $878 \mathrm{a}$ & $4,5 \mathrm{a}$ & $3,8 \mathrm{ab}$ & $7,7 \mathrm{a}$ & $6,5 \mathrm{a}$ \\
\hline & IT & $97 a$ & $91 \mathrm{a}$ & $778 \mathrm{a}$ & $903 a$ & $4,2 \mathrm{ab}$ & $6,3 \mathrm{a}$ & $5,3 \mathrm{a}$ & $5,6 \mathrm{a}$ \\
\hline & IFT & $79 b$ & $90 a^{*}$ & $866 a$ & $1029 a$ & $5,5 \mathrm{a}$ & $6,3 a$ & $8,3 \mathrm{a}$ & $8,0 \mathrm{a}$ \\
\hline & $\mathrm{C}$ & $0 \mathrm{c}$ & $0 \mathrm{~b}$ & $226 b$ & $283 b$ & $1,1 \mathrm{~b}$ & $1,7 \mathrm{~b}$ & $1,2 b$ & $1,8 \mathrm{~b}$ \\
\hline \multirow[t]{4}{*}{ Sesbânia } & IF & $98 a^{*}$ & $75 a$ & $190 \mathrm{a}$ & $315 a b^{*}$ & $17,7 \mathrm{a}$ & $24,8 a^{*}$ & $9,4 a$ & $11,7 \mathrm{a}$ \\
\hline & IT & $98 a^{*}$ & $83 a$ & $248 \mathrm{a}$ & $225 b$ & $16,0 \mathrm{a}$ & $30,9 a^{*}$ & $8,3 \mathrm{a}$ & $13,4 a^{*}$ \\
\hline & IFT & $96 a$ & $87 a$ & $243 a$ & $376 a *$ & $16,6 a$ & $25,1 \mathrm{a}^{*}$ & $9,3 \mathrm{a}$ & $12,2 \mathrm{a}$ \\
\hline & $\mathrm{C}$ & $0 \mathrm{~b}$ & $0 \mathrm{~b}$ & $31 b$ & $220 b *$ & $8,0 \mathrm{~b}$ & $21,6 a^{*}$ & $4,0 \mathrm{~b}$ & $10,0 a^{*}$ \\
\hline \multirow[t]{4}{*}{ Tamboril } & IF & $96 a^{*}$ & $82 a$ & $151 \mathrm{a}$ & $268 b^{*}$ & $5,2 \mathrm{ab}$ & $6,4 \mathrm{ab}$ & $23,3 a^{*}$ & $14,2 \mathrm{~b}$ \\
\hline & IT & $88 \mathrm{a}$ & $79 a$ & $235 \mathrm{a}$ & $494 a^{*}$ & $4,0 \mathrm{~b}$ & $4,8 b$ & $2,8 \mathrm{c}$ & $19,8 \mathrm{a}$ \\
\hline & IFT & $93 a$ & $92 \mathrm{a}$ & $241 \mathrm{a}$ & $326 b$ & $6,7 \mathrm{a}$ & $7,7 \mathrm{a}$ & $13,1 \mathrm{~b}$ & $11,7 \mathrm{c}$ \\
\hline & $\mathrm{C}$ & $0 \mathrm{~b}$ & $0 b$ & $25 b$ & $28 \mathrm{c}$ & $0,9 \mathrm{c}$ & $1,6 \mathrm{c}$ & $1,7 \mathrm{c}$ & $2,5 \mathrm{~d}$ \\
\hline
\end{tabular}

1 Médias seguidas da mesma letra não diferem entre si em tratamentos de inoculação, em cada espécie, pelo teste de Tukey a 5\% de probabilidade.

2 IF: plantas com inoculação na formação das mudas; IT: plantas com inoculação no transplante; IFT: plantas com inoculação nas duas épocas; C: plantas sem nenhuma inoculação.

* Efeito significativo de doses de fertilização a 5\% de probabilidade. 
micorrização para a produção de massa de raízes em condições subótimas de fertilidade. $\mathrm{O}$ aumento da fertilização resultou em vantagens ao crescimento das raízes nas plantas não-micorrizadas de açoitacavalo, embaúba e sesbânia. Na cássia-verrugosa e sesbânia, a massa seca de raízes foi influenciada pela dose de nutrientes, mas a inoculação das plantas IF no transplantio não teve efeito significativo.

Os efeitos dos tratamentos na nodulação foram mais notáveis na sesbânia, que apresentou nodulação mais abundante (Tabela 4). Mudas das duas espécies sem inoculação de FMAs apresentaram nodulação muito reduzida, mesmo na maior dose de PK. Salienta-se a baixa nodulação do tamboril, o que não ocorreu em outro estudo com esta espécie, no mesmo solo (Moreira \& Siqueira, 1995). Talvez as condições nutricionais de formação das mudas tenham sido inadequadas para a nodulação do tamboril. A atividade da nitrogenase foi também influenciada pelos tratamentos de inoculação e fertilização nas duas espécies, sendo superior no tamboril, quando recebeu o inóculo na formação, e transplantado em solo de alta fertilidade. Portanto, o tamboril produziu menor massa nodular, porém exibiu elevada atividade específica da nitrogenase, em comparação com sesbânia, especialmente nas plantas com inoculação de FMAs. Desse modo, tanto a micorrização quanto a elevação da fertilidade pode contribuir para elevar a capacidade de fixar o $\mathrm{N}_{2}$ atmosfé- rico destas espécies, como tem sido relatado por vários autores, revisado em Barea et al. (1992). Isto reflete em melhoria nutricional e crescimento das plantas, o que se torna de grande importância para o reflorestamento em solos de baixa fertilidade.

Os efeitos relativos dos tratamentos de inoculação das várias espécies estudadas encontram-se na Fig. 1. A introdução do inóculo nas mudas em formação (IF/C) aumentou a produção de MS em apenas $15 \%$ na sesbânia, $500 \%$ na cássia-verrugosa e $800 \%$ na colvílea em alta fertilização, ao passo que na inoculação no transplantio a produção de MS atingiu um máximo de $400 \%$. A colvílea não respondeu positivamente à inoculação no transplantio, e esta teve efeito depressivo e acentuado quando expresso pela relação IT/IF. Como verificado também em relação a outros parâmetros, conclui-se que para garantir desenvolvimento adequado desta espécie após o transplantio, a mesma deve ser submetida a inoculação no período da formação das mudas. As demais espécies comportaram-se diferentemente desta, podendo a inoculação ser feita no período de formação ou no transplantio. A condição micorrízica das mudas e o potencial de inóculo do solo onde estas serão transplantadas exerceram efeitos acentuados em seu crescimento após o transplantio. A inoculação em mudas já micorrizadas no transplantio resultou em algum efeito positivo no fedegoso, na sesbânia, na embaúba e no açoita-cavalo, porém este

TABELA4. Nodulação e atividade da nitrogenase em nódulos frescos em sesbânia e tamboril sob tratamentos de inoculação e doses baixa $\left(D_{1}\right)$ e alta $\left(D_{2}\right)$ de nutrientes aplicados no solo'.

\begin{tabular}{|c|c|c|c|c|c|c|c|}
\hline \multirow[t]{2}{*}{ Espécie } & \multirow[t]{2}{*}{ Tratamento $^{2}$} & \multicolumn{2}{|c|}{ Número de nódulos } & \multicolumn{2}{|c|}{ Peso de nódulos, secos } & \multicolumn{2}{|c|}{ Atividade da nitrogenase } \\
\hline & & $D_{1}$ & $\mathrm{D}_{2}$ & $\mathrm{D}_{1}$ & $\mathrm{D}_{2}$ & $\mathrm{D}_{1}$ & $\mathrm{D}_{2}$ \\
\hline & & $\begin{array}{ll}-----(1 \\
-1\end{array}$ & $\begin{array}{l}1 \\
\end{array}-----$ & ------ $(\mathrm{g}$ & ------ & $\left(\mu\right.$ moles $C_{2}$ & $\left.u^{\prime o s}{ }^{-1} h^{-1}\right)$ \\
\hline \multirow[t]{4}{*}{ Tamboril } & IF & $11 \mathrm{a}$ & $27 a^{*}$ & $0,05 \mathrm{a}$ & $0,31 \mathrm{a}^{*}$ & $1,4 a b$ & $7,9 a^{*}$ \\
\hline & IT & $8 b$ & $11 c^{*}$ & $0,02 \mathrm{a}$ & $0,13 b^{*}$ & $1,0 \mathrm{ab}$ & $1,0 \mathrm{c}$ \\
\hline & IFT & $8 b$ & $19 b^{*}$ & $0,04 \mathrm{a}$ & $0,17 b^{*}$ & $2,1 \mathrm{a}$ & $3,2 b$ \\
\hline & $\mathrm{C}$ & $3 \mathrm{c}$ & $8 \mathrm{~d}^{*}$ & $0,02 \mathrm{a}$ & $0,03 \mathrm{c}$ & $0,3 b$ & $1,3 b c$ \\
\hline \multirow[t]{4}{*}{ Sesbânia } & IF & $63 \mathrm{a}$ & $213 a^{*}$ & $0,8 \mathrm{a}$ & $1,3 b$ & $1,6 \mathrm{a}$ & $2,0 \mathrm{bc}$ \\
\hline & IT & $38 b$ & $116 b^{*}$ & $0,5 \mathrm{a}$ & $1,0 \mathrm{~b}$ & $0,8 \mathrm{~b}$ & $4,8 \mathrm{a}^{*}$ \\
\hline & IFT & $39 b$ & $187 a^{*}$ & $0,8 \mathrm{a}$ & $2,3 a^{*}$ & $1,2 \mathrm{ab}$ & $3,2 b^{*}$ \\
\hline & $\mathrm{C}$ & $1 \mathrm{c}$ & $33 c$ & $0,01 \mathrm{~b}$ & $0,7 \mathrm{~b} *$ & $0,2 \mathrm{~b}$ & $0,4 \mathrm{c}$ \\
\hline
\end{tabular}

1 Médias seguidas da mesma letra não diferem entre si em tratamentos de inoculação, em cada espécie, pelo teste de Tukey a 5\% de probabilidade

2 IF: plantas com inoculação na formação das mudas; IT: plantas com inoculação no transplante; IFT: plantas com inoculação nas duas épocas; C: plantas sem nenhuma inoculação.

* Efeito significativo de doses de fertilização a $5 \%$ de probabilidade 
foi pequeno, o que indica ser um procedimento desnecessário. Isto sugere que, tratando-se de mudas submetidas a inoculação no período da formação, a condição micorrízica do solo para onde estas mudas serão transplantadas terá pouca influência no crescimento após o transplantio. As razões pelas quais as espécies apresentam comportamento distinto em relação à micorrização e a fertilidade do solo são ainda desconhecidas. No entanto, aspectos como a de-

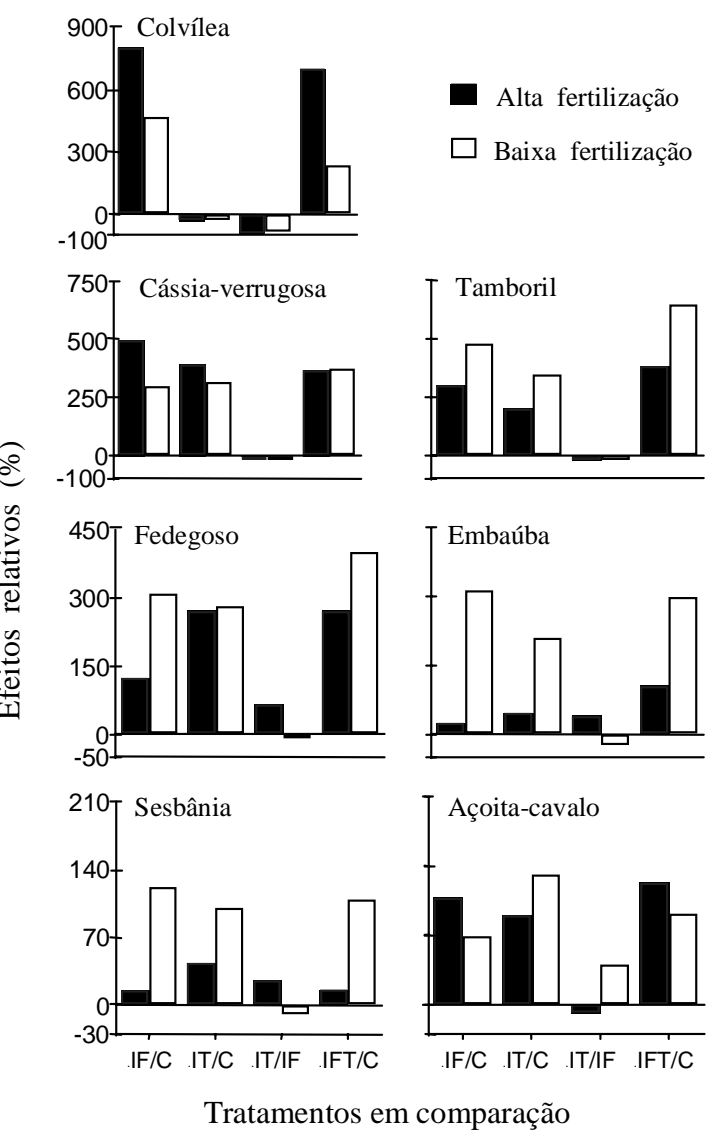

FIG. 1. Efeitos relativos da inoculação em diferentes épocas, na produção de matéria seca da parte aérea de sete espécies florestais. IF/C- efeito relativo da inoculação na formação (IF) sobre o controle; IT/C- efeito relativo da inoculação no transplantio sobre o controle; IT/IF- efeito de inoculação no transplante sobre a inoculação na formação; IFT/C- efeito de inoculação nas duas épocas sobre $C$. manda de nutrientes e as relações entre a capacidade fotossintética da planta e o dreno de fotoassimilados causados pela micorrização, modulados pelo seu estado nutricional, poderão explicar as respostas aqui obtidas, merecendo, portanto, estudos.

\section{Nutrientes na matéria seca da parte aérea das plantas}

Os efeitos dos tratamentos estudados sobre os teores dos nutrientes variaram muito; os nutrientes mais influenciados são apresentados na Tabela 5. O teor de $\mathrm{N}$ não foi influenciado pelos tratamentos de inoculação no açoita-cavalo, embaúba e no tamboril na dose baixa de nutrientes. A embaúba mostrou teores mais elevados de $\mathrm{N}$, na dose mais baixa de NPK, onde houve menor crescimento, o que indica efeito de diluição do nutriente (Jarrel \& Beverly, 1981) na matéria seca das plantas na dose alta de NPK. A inoculação na fase da formação aumentou os teores de $\mathrm{N}$ no tamboril e na sesbânia, em alta fertilização com PK. Como não foi aplicado N no solo, nestas espécies, existindo deficiência deste nutriente, a inoculação favoreceu a fixação do N, como já foi discutido (Tabela 4). Entretanto, os efeitos da micorriza na maior absorção do $\mathrm{N}$ do solo por mudas de espécies de árvores nativas (Pereira et al., 1996) não pode ser descartado. $\mathrm{O}$ fato é que a inoculação favoreceu a nutrição nitrogenada destas duas espécies. Os efeitos dos tratamentos nos teores de P são mais constantes do que o observado em relação a outros nutrientes (Tabela 5). A embaúba, o açoitacavalo e a sesbânia exibiram teores maiores deste nutriente do que as outras espécies. A elevação por ocasião da fertilização aumentou o teor de P em todas as espécies, exceto no tamboril, em que não houve nenhum efeito, e no fedegoso, em que houve redução nos teores deste nutriente nas plantas com inoculação. Houve efeito da inoculação em açoitacavalo, fedegoso, na cássia-verrugosa e embaúba, especialmente na fertilização mais elevada. $\mathrm{Na}$ ausência de micorriza, apenas a embaúba apresentou teor de P aumentado pela elevação de NPK. Portanto é evidente a importância das micorrizas para a absorção de $\mathrm{P}$ por mudas da maioria das espécies de árvores tropicais estudadas (Carneiro et al., 1996; Saggin Júnior, 1997; Siqueira et al., 1998). Tal como ocorreu 
com relação ao N, houve tendência de efeito de diluição do teor de P na planta (Jarrel \& Beverly, 1981).

Os teores de $\mathrm{K}$ na parte aérea das mudas das espécies foram diferentemente influenciados pelos tratamentos (Tabela 5). A elevação da adubação exerceu efeitos em poucas espécies e tratamentos, sendo este, pouco consistentes. Em baixa fertilização, a micorrização teve efeito positivo no teor de $\mathrm{K}$ no açoita-cavalo, na colvílea e na embaúba, diminuiu no tamboril e não teve efeito nas demais espécies. Em alta fertilização estes efeitos foram mais raros e inconsistentes. No que tange ao $\mathrm{Ca}$, verificou-se efeito benéfico da micorrização em várias espécies e da elevação da fertilidade, especialmente em plantas sem inoculação. Portanto, como várias destas espécies absorvem grande quantidade de $\mathrm{Ca}$ (Carneiro et al.,

TABELA5. Teores de N, P, K, Ca e Zn na parte aérea de espécies arbóreas sob tratamentos de inoculação e doses baixa $\left(D_{1}\right)$ e alta $\left(D_{2}\right)$ de nutrientes aplicados no solo ${ }^{1}$.

\begin{tabular}{|c|c|c|c|c|c|c|c|c|c|c|c|}
\hline \multirow[t]{2}{*}{ Espécie } & \multirow{2}{*}{$\begin{array}{l}\text { Trata- } \\
\text { mento }^{2}\end{array}$} & \multicolumn{2}{|c|}{$\mathrm{N}$} & \multicolumn{2}{|c|}{$\mathrm{P}$} & \multicolumn{2}{|c|}{$\mathrm{K}$} & \multicolumn{2}{|c|}{$\mathrm{Ca}$} & \multicolumn{2}{|c|}{$\mathrm{Zn}$} \\
\hline & & $\mathrm{D}_{1}$ & $\mathrm{D}_{2}$ & $\mathrm{D}_{1}$ & $\mathrm{D}_{2}$ & $\mathrm{D}_{1}$ & $\mathrm{D}_{2}$ & $D_{1}$ & $\mathrm{D}_{2}$ & $D_{1}$ & $\mathrm{D}_{2}$ \\
\hline & & \multicolumn{2}{|c|}{---- $\left(\mathrm{g} \mathrm{kg}^{-1}\right)$---- } & \multicolumn{2}{|c|}{---- $\left(\mathrm{g} \mathrm{kg}^{-1}\right)$---- } & \multicolumn{2}{|c|}{--- $\left(\mathrm{g} \mathrm{kg}^{-1}\right)$---- } & \multicolumn{2}{|c|}{---- $\left(\mathrm{g} \mathrm{kg}^{-1}\right)$---- } & \multicolumn{2}{|c|}{--- $\left(\mathrm{mg} \mathrm{kg}^{-1}\right)$---- } \\
\hline \multirow[t]{4}{*}{ Açoita-cavalo } & IF & $38,7 \mathrm{a}$ & $34,6 a$ & $2,50 \mathrm{a}$ & $3,44 a^{*}$ & $24,2 \mathrm{a}$ & $20,7 \mathrm{a}$ & $26,7 \mathrm{ab}$ & $26,7 \mathrm{a}$ & $62,6 b$ & $59,8 \mathrm{bc}$ \\
\hline & IT & $35,6 a$ & $33,6 a$ & $2,22 \mathrm{a}$ & $3,41 a^{*}$ & $24,2 \mathrm{a}$ & $22,6 a$ & $30,9 a$ & $27,6 \mathrm{a}$ & $83,7 \mathrm{a}$ & 78,7ab \\
\hline & IFT & $35,9 a$ & $37,9 a$ & $1,84 a b$ & $3,44 a^{*}$ & 19,9ab & $21,8 \mathrm{a}$ & $26,4 a b$ & $31,1 a^{*}$ & $54,7 \mathrm{~b}$ & $80,0 a^{*}$ \\
\hline & $\mathrm{C}$ & $38,4 \mathrm{a}$ & $41,4 \mathrm{a}$ & $1,29 b$ & $1,55 b$ & $15,6 b$ & $19,4 \mathrm{a}$ & $21,2 \mathrm{~b}$ & $28,5 a^{*}$ & $71,8 \mathrm{ab} *$ & $56,9 \mathrm{c}$ \\
\hline \multirow[t]{4}{*}{ C.-verrugosa } & IF & $34,5 a b^{*}$ & $25,2 b$ & $1,50 \mathrm{a}$ & $2,10 a b^{*}$ & $10,7 \mathrm{a}$ & $10,2 \mathrm{a}$ & $36,2 \mathrm{a}$ & $35,5 \mathrm{a}$ & $35,2 \mathrm{a}$ & $33,2 \mathrm{a}$ \\
\hline & IT & $31,1 \mathrm{~b}$ & $28,5 b$ & $1,40 \mathrm{a}$ & $2,20 a^{*}$ & $10,6 a$ & $11,2 \mathrm{a}$ & $36,7 \mathrm{a}$ & $38,1 \mathrm{a}$ & $29,8 \mathrm{a}$ & $38,5 \mathrm{a}$ \\
\hline & IFT & $30,8 b$ & $29,4 b$ & $1,30 \mathrm{a}$ & $1,90 \mathrm{ab} *$ & $9,7 \mathrm{a}$ & $11,4 a^{*}$ & $35,9 a$ & $38,1 \mathrm{a}$ & $30,1 \mathrm{a}$ & $31,1 \mathrm{a}$ \\
\hline & $\mathrm{C}$ & $41,2 \mathrm{a}$ & $38,2 \mathrm{a}$ & $1,20 \mathrm{a}$ & $1,40 \mathrm{~b}$ & $11,7 \mathrm{a}$ & $10,4 \mathrm{a}$ & $16,5 \mathrm{a}$ & $28,0 a^{*}$ & $23,8 \mathrm{a}$ & $33,4 a^{*}$ \\
\hline \multirow[t]{4}{*}{ Colvílea } & IF & $65,2 \mathrm{ab} *$ & $49,2 \mathrm{a}$ & $1,93 b$ & $1,51 \mathrm{a}$ & $35,4 a^{*}$ & $25,6 \mathrm{c}$ & $18,0 a^{*}$ & $13,6 \mathrm{a}$ & $71,7 \mathrm{a}$ & $57,0 \mathrm{a}$ \\
\hline & IT & $75,3 a^{*}$ & $55,2 \mathrm{ab}$ & $1,94 b$ & $1,53 \mathrm{a}$ & $32,4 a^{*}$ & $25,3 \mathrm{c}$ & $15,8 \mathrm{ab} *$ & $6,6 b$ & $60,2 \mathrm{a}$ & $46,0 \mathrm{a}$ \\
\hline & IFT & $78,5 \mathrm{a}^{*}$ & $65,2 \mathrm{ab}$ & $1,46 b$ & $2,04 a^{*}$ & $25,4 b$ & $30,0 b^{*}$ & $12,8 \mathrm{bc}$ & $16,5 \mathrm{a}$ & $43,9 \mathrm{a}$ & $58,7 \mathrm{a}$ \\
\hline & $\mathrm{C}$ & $58,0 \mathrm{~b}$ & $71,2 a^{*}$ & $2,69 a^{*}$ & $2,04 \mathrm{a}$ & $25,3 b$ & $35,9 a^{*}$ & $8,2 \mathrm{c}$ & $17,4 a^{*}$ & $42,1 \mathrm{a}$ & $63,4 a$ \\
\hline \multirow[t]{4}{*}{ Embaúba } & IF & $42,7 a^{*}$ & $38,2 \mathrm{a}$ & $2,00 \mathrm{a}$ & $3,05 \mathrm{a}^{*}$ & $26,0 \mathrm{~b}$ & $30,6 b$ & $38,1 \mathrm{ab}$ & $32,7 \mathrm{ab}$ & $35,2 \mathrm{a}$ & $33,2 \mathrm{a}$ \\
\hline & IT & $42,8 a^{*}$ & $39,8 \mathrm{a}$ & $2,42 \mathrm{a}$ & $3,07 \mathrm{a}^{*}$ & $40,1 a^{*}$ & $34,8 \mathrm{a}$ & $42,7 \mathrm{a}$ & $38,2 \mathrm{ab}$ & $29,8 \mathrm{a}$ & $38,5 a^{*}$ \\
\hline & IFT & $44,0 a^{*}$ & $39,9 a$ & $1,86 \mathrm{~b}$ & $3,20 a^{*}$ & $29,6 \mathrm{~b}$ & $34,5 b$ & $38,5 \mathrm{ab}$ & $45,5 \mathrm{a}$ & $30,1 \mathrm{a}$ & $31,1 \mathrm{a}$ \\
\hline & $\mathrm{C}$ & $47,3 a^{*}$ & $40,9 a$ & $1,28 b$ & $1,96 b^{*}$ & $31,0 \mathrm{~b}$ & $41,2 a^{*}$ & $25,6 \mathrm{~b}$ & $30,6 b$ & $28,9 a$ & $33,4 \mathrm{a}$ \\
\hline \multirow[t]{4}{*}{ Fedegoso } & IF & $33,4 b$ & $27,0 \mathrm{~b}$ & $2,43 a^{*}$ & $1,77 \mathrm{a}$ & $21,1 a^{*}$ & $15,4 \mathrm{a}$ & $39,3 \mathrm{a}$ & $34,8 \mathrm{ab}$ & $31,1 \mathrm{a}$ & $37,7 a^{*}$ \\
\hline & IT & $36,3 b$ & $33,9 b$ & $2,34 a^{*}$ & $1,80 \mathrm{a}$ & $19,4 a^{*}$ & $16,0 \mathrm{a}$ & $43,7 a^{*}$ & $37,7 \mathrm{a}$ & $32,5 \mathrm{a}$ & $36,0 \mathrm{a}$ \\
\hline & IFT & $35,1 \mathrm{~b}$ & $28,2 b$ & $2,27 \mathrm{a}^{*}$ & $1,68 \mathrm{a}$ & $18,8 a^{*}$ & $15,4 \mathrm{a}$ & $41,7 a^{*}$ & $36,5 a$ & $31,9 a$ & $33,5 a$ \\
\hline & $\mathrm{C}$ & $48,5 \mathrm{a}$ & $46,2 \mathrm{a}$ & $1,16 b$ & $1,07 \mathrm{~b}$ & $19,2 \mathrm{a}$ & $16,5 \mathrm{a}$ & $28,5 b$ & $28,9 b$ & $32,3 a^{*}$ & $23,2 b$ \\
\hline \multirow[t]{4}{*}{ Sesbânia } & IF & $51,1 \mathrm{bc}$ & $50,5 \mathrm{a}$ & $2,10 \mathrm{c}$ & $2,90 a^{*}$ & $19,1 \mathrm{a}$ & $19,0 \mathrm{a}$ & $20,7 \mathrm{a}$ & $19,3 b$ & $54,5 \mathrm{a}$ & $53,2 \mathrm{a}$ \\
\hline & IT & $57,5 \mathrm{a}^{*}$ & $48,9 \mathrm{a}$ & $3,10 \mathrm{a}$ & $2,80 \mathrm{a}$ & $20,0 a^{*}$ & $17,1 \mathrm{a}$ & $18,6 \mathrm{a}$ & $19,8 \mathrm{~b}$ & $62,7 a^{*}$ & $53,8 \mathrm{a}$ \\
\hline & IFT & $48,9 \mathrm{c}$ & $50,7 \mathrm{a}$ & $2,40 b c$ & $2,50 \mathrm{ab}$ & $19,6 \mathrm{a}$ & $18,5 \mathrm{a}$ & $19,9 a$ & $20,8 b$ & $53,6 \mathrm{a}$ & $52,5 \mathrm{a}$ \\
\hline & $\mathrm{C}$ & $60,5 \mathrm{a}^{*}$ & $52,0 \mathrm{~b}$ & $2,80 \mathrm{ab} *$ & $2,30 \mathrm{~b}$ & $17,7 \mathrm{a}$ & $19,0 \mathrm{a}$ & $12,6 b$ & $26,9 a^{*}$ & $39,9 b$ & $50,8 a^{*}$ \\
\hline \multirow[t]{4}{*}{ Tamboril } & IF & $26,7 \mathrm{a}$ & $42,6 a^{*}$ & $1,05 \mathrm{ab}$ & $0,99 \mathrm{a}$ & $11,9 b$ & $17,6 a^{*}$ & $16,1 \mathrm{a}$ & $15,9 \mathrm{a}$ & $42,9 b$ & $53,4 \mathrm{a}$ \\
\hline & IT & $31,3 \mathrm{a}$ & $30,8 b$ & $0,79 b$ & $1,08 \mathrm{a}$ & $12,7 b$ & $14,9 \mathrm{ab}$ & $17,9 a^{*}$ & $15,1 \mathrm{a}$ & $53,2 \mathrm{~b}$ & $48,8 \mathrm{a}$ \\
\hline & IFT & $31,1 \mathrm{a}$ & $28,9 b$ & $1,66 \mathrm{a}$ & $1,51 \mathrm{a}$ & $13,4 a b$ & $13,9 b$ & $16,7 \mathrm{a}$ & $16,3 \mathrm{a}$ & $45,4 b$ & $51,5 \mathrm{a}$ \\
\hline & $\mathrm{C}$ & $28,8 \mathrm{a}$ & $27,4 \mathrm{~b}$ & $1,23 \mathrm{ab}$ & $1,50 \mathrm{a}$ & $16,4 a^{*}$ & $13,2 b$ & $17,9 a^{*}$ & $15,7 \mathrm{a}$ & $48,1 a^{*}$ & $38,8 \mathrm{a}$ \\
\hline
\end{tabular}

1 Médias seguidas da mesma letra não diferem entre si em tratamentos de inoculação, em cada espécie, pelo teste de Tukey a 5\% de probabilidade.

2 IF: plantas com inoculação na formação das mudas; IT: plantas com inoculação no transplante; IFT: plantas com inoculação nas duas épocas; C: plantas sem nenhuma inoculação.

* Efeito significativo de doses de fertilização a $5 \%$ de probabilidade. 
1996) a contribuição das micorrizas na melhoria da nutrição deste elemento, não deve ser desprezada, nas condições tropicais. A inoculação favoreceu os teores de Zn em açoita-cavalo, fedegoso e sesbânia, e diminui no tamboril, porém de modo pouco consistente; o mesmo ocorreu quanto aos efeitos da adubação. A inconsistência destes efeitos deve resultar das interações entre $P$, Zn e micorrizas que certamente varia entre as espécies.

Todas as espécies responderam positivamente à inoculação em termos de desenvolvimento após o transplante. Verifica-se, portanto, que a condição micorrízica da muda e a presença ou ausência de propágulos de FMAs no solo de plantio, e não apenas o nível de fertilidade, são fatores que modulam o desenvolvimento pós-transplante de mudas das espécies estudadas. No açoita-cavalo, fedegoso, cássia-verrugosa e embaúba, os benefícios da micorrização relacionaram-se com efeitos nutricionais e teores mais elevados de $\mathrm{P}$, corroborando outros resultados com estas espécies (Carneiro et al., 1996; Saggin Júnior, 1997; Siqueira et al., 1998). A colvílea manifestou enorme dependência em relação à inoculação em mudas no período de formação, e, ao contrário do que ocorreu para as demais espécies, não apresentou resposta à inoculação no transplantio, mesmo apresentando teores elevados de $\mathrm{K}$ e Ca neste tratamento. Isto sugere a existência de mecanismos diferenciados de respostas destas espécies à micorrização.

\section{CONCLUSÕES}

1. As espécies estudadas apresentam elevada colonização micorrízica, quando submetidas a inoculação tanto durante a formação como no momento do transplante das mudas.

2. As respostas em crescimento devido à micorrização são diferenciadas pelas épocas de inoculação, sendo que todas as espécies respondem positivamente à inoculação na formação e no transplantio das mudas, exceto a colvílea, que não se beneficia da inoculação no transplantio.

3. Não há benefício adicional na inoculação no transplantio de mudas já submetidas a inoculação durante a formação; entretanto, esta última pode ser uma alternativa para garantir o desenvolvimento de mudas sem inoculação ou com baixa formação de micorrizas na formação.

4. Os efeitos da micorrização no crescimento das mudas após o transplantio são mediados pelos efeitos da simbiose na nutrição, e, portanto, relacionados à fertilização do solo.

\section{REFERÊNCIAS}

BAREA, J.M.; AZCÓN, R.; AZCÓN AGUILAR, C Vesicular-arbuscular mycorrhizal fungi in nitrogenfixing system. In: NORRIS, J.R.; READ, D.J.; VARMA, A.K. (Eds.). Methods in microbiology London : Academic, 1992. v.24, p.391-416.

BLANCHAR, R.W.; REHM, G.; CALDWELL, A.C. Sulfur in plant material digestion with nitric and percloric acid. Soil Science Society of America. Proceedings, Madison, v.29, n.1, p.71-72, Jan. 1965.

BREMNER, J.M.; EDWARD, H.P. Determination and isotope ratio analysis of different from of nitrogen in soils. I. Apparatus and procedures for destination and determination for ammonium. Soil Science Society of America. Proceedings, Madison, v.29, n.5, p.504-507, Sept. 1965

CARNEIRO, M.A.C.; SIQUEIRA, J.O.; DAVIDE, A.C.; GOMES, L.J.; CURI, N.; VALE, F.R. do. Fungo micorrízico e superfosfato no crescimento de espécies arbóreas tropicais. Scientia Forestalis Piracicaba, v.50, p.21-36. dez. 1996

DAVIDE, A.C.; FARIA, J.M.R.; BOTELHO, S.A. Propagação de espécies florestais. Belo Horizonte CEMIG/UFLA/FAEPE, 1995. 40p.

GIOVANNETTI, M.; MOSSE, B. An evaluation of techniques to measure vesicular-arbuscular infection in roots. The New Phytologist, London, v.84, n.4, p.489-500, Apr. 1980

HERRERA, R.A.; RODRÍGUEZ, M.E.; OROZCO, M.O.; FURRAZOLA, E; FERRER, R.L. Las micorrizas y el funcionamiento de los bosques tropicales. In: HERRERA, R.; MENÉNDEZ, L.; RODRIGUEZ, M.E.; GARCÍA, E.E. (Eds.). Ecología de los Bosques Siempreverdes de la Sierra del Rosario, Cuba: 1974-1987. La Habana : Instituto de Ecología y Sistemática/Academia de Ciencias de Cuba, 1988. p.296-323. (Proyecto MAB, 1).

JANOS, D.P. Mycorrhizas, succession and the rehabilitation of deforested lands in the humid tropics. In: FRANKLAND, J.C.; MAGAN, N.; 
GADD, G.M. (Eds.). Fungi and environmental change : British Mycological Society Symposium. Cambridge, UK : Cambridge University Press, 1996. v. 20, p. $129-162$

JARREL, W.M.; BEVERLY, R.B. The dilution effect in plant nutrition studies. Advances in Agronomy, New York, v.34, p.197-224, 1981

LIAO, C.F.H. Devarda's alloy method for total nitrogen determination. Soil Science Society of America. Journal, Madison, v.45, n.5, p.852-855, Sept./Oct 1981

LORENZI, H. Árvores brasileiras: manual de identificação e cultivo de plantas arbóreas nativas do Brasil. Nova Odessa : Plantarum, 1992. 352p.

MOREIRA, F.M.; SIQUEIRA, J.O. Growth, nodulation and arbuscular mycorrhizal colonization of four woody legumes in a low-fertility soil. In: INTERNATIONAL SYMPOSIUM ON SUSTAINABLE AGRICULTURE FOR THE TROPICS - THE ROLE OF BIOLOGICAL NITROGEN FIXATION, 1995, Angra dos Reis, RJ Anais. Angra dos Reis : Embrapa-CNPAB/UFRRJ, 1995. p.164-165.

PEREIRA, E.G.; SIQUEIRA, J.O.; CURI, N.; MOREIRA F.M.; PURCINO, A.A.C. Efeitos da micorriza e do suprimento de fósforo na atividade enzimática e na resposta de espécies arbóreas ao nitrogênio. Revista Brasileira de Fisiologia Vegetal, Brasília, v. 8 n.1, p.59-66, jan./abr. 1996

PHILLIPS, J.M.; HAYMAN, D.S. Improved procedures for clearing roots and staining parasitic and vesiculararbuscular mycorrhizal fungi for rapid assessment of infection, Transactions of the British Mycological Society, London, v.55, p.158-161, Aug. 1970

RAIJ, B. van. Fertilidade do solo e adubação. São Paulo : Agronomia Ceres, 1991. 343p.

SAGGIN JÚNIOR, O.J. Micorrizas arbusculares em mudas de espécies arbóreas nativas do sudeste brasileiro. Lavras : UFLA, 1997. 120p. Tese de Doutorado.

SAGGIN JÚNIOR, O.J.; SIQUEIRA, J.O.; COLOZZIFILHO, A.; OLIVEIRA, E. A infestação do solo com fungos micorrízicos no crescimento pós-transplante de mudas de cafeeiro não micorrizadas. Revista Brasileira de Ciências do Solo, Campinas, v.16, n.1, p.39-46, 1992

SAGGIN JÚNIOR, O.J.; SIQUEIRA, J.O.; GUIMARÃES, P.T.G.; OLIVEIRA, E. Interação fungos micorrízicos versus superfosfato e seus efeitos no crescimento e teores de nutrientes do cafeeiro em solo não fumigado. Revista Brasileira de Ciências do Solo, Campinas, v.18, n.1, p.27-36, jan./fev. 1994.

SIQUEIRA, J.O.; CARNEIRO, M.A.C.; CURI, N.; SILVA, S.C. da; DAVIDE, A.C. Mycorrhizal colonization and mycotrophic growth of native woody species as related to sucessional groups in Southeastern Brasil. Forest Ecology and Management, Amsterdam, v. 107, p.241-252, 1998.

SIQUEIRA, J.O.; COLOZZI FILHO, A.; SAGGIN JUNIOR, O.J.; GUIMARÃES, P.T.G.; OLIVEIRA, E. Crescimento de mudas e produção do cafeeiro sob influência de fungos micorrízicos e superfosfato. Revista Brasileira de Ciência do Solo, Campinas, v.17, n.1, p.53-60. jan./abr. 1993.

SIQUEIRA, J.O.; MOREIRA, F.M.; GRISI, B.M.; HUNGRIA, M.; ARAUJO, R. Microrganismos e processos biológicos do solo: perspectiva ambiental. Brasília : Embrapa-SPI, 1994. 142p. (Embrapa-CNPAF. Documentos, 5).

SIQUEIRA, J.O.; SAGGIN JÚNIOR, O.J. The importance of mycorrhizae association in natural low-fertility soils. In: INTERNATIONAL SYMPOSIUM ON ENVIROMENTAL STRESS, 1., 1992, Belo Horizonte. Maize in perspective: proceedings. Sete Lagoas : Embrapa-CNPMS/México : CIMMYT/ UNDP, 1995. p.239-280

SIQUEIRA, J.O.; SAGGIN JUNIOR, O.J.; COLOZZI FILHO, A.; OLIVEIRA, E. Influência do substrato de formação e da micorriza no crescimento de mudas de cafeeiro transplantadas. Pesquisa Agropecuária Brasileira, Brasília, v.30, n.12, p.1417-1425, dez 1995.

VETTORI, L. Métodos de análises do solo. Rio de Janeiro : Ministério da Agricultura, 1969. 24p. (Boletim técnico, 7)

ZAROSKY, R.J.; BURAU, R.G. A rapid nitric perchloric acid digestion method for multi-element tissue analysis. Communications in Soil Science and Plant Analysis, New York, v.8, n.5, p.425-436, 1977.

ZONTA, E.P.; MACHADO, A.A.; SILVEIRA JUNIOR, P. Sistemas de Análise Estatística para Microcomputadores (SANEST). Pelotas : UFPel, Departamento de Matemática e Estatística, 1984 $151 \mathrm{p}$. 